\title{
Expansão agropecuária: o efeito sobre a vegetação e o microclima
}

\section{Agriculture and livestock expansion: the effect on vegetation and microclimate}

\author{
Nathan Castro Fonsêca $\mathbb{1}^{1 *}$, Jéssica Stéfane Alves da Cunha $\mathbb{D}^{1}$, José Alberes Santos da Cunha ${ }^{1}$, Lúcia \\ dos Santos Rodrigues ${ }^{1}{ }^{1}$, Isabela Souto Maior Belém (D) $^{2}$
}

${ }^{1}$ Programa de Pós-Graduação em Ciências Florestais, Universidade Federal Rural de Pernambuco, Recife, Pernambuco; (81) 982587967, nathanflorestal@hotmail.com; cunhajsa@gmail.com; alberesask122@gmail.com; lucia.ufal@gmail.com. ${ }^{2}$ Departamento de Biologia, Universidade Federal Rural de Pernambuco, Recife, Pernambuco., belasoutomaior@gmail.com.

\section{A R T I G O}

Recebido: 11/06/2018

Aprovado: 03/09/2019

\section{Palavras-chave: \\ Bem-estar humano \\ Mudanças climáticas \\ Desmatamento}

\section{Key words:}

Human well-being

Climate changes

Deforestation

\begin{abstract}
R E S U M O
A pressão sobre os recursos naturais para fins de atividades agropecuárias tem causado alterações e perdas de serviços ecossistêmicos essenciais ao bem-estar das populações. Objetivou-se avaliar a influência do desmatamento sob as alterações climáticas em áreas de exploração agropecuária do município de Currais-PI. Realizou-se uma análise espaço-temporal do uso e cobertura do solo no município, numa serie temporal de 32 anos (1986 a 2018), correlacionando com variáveis meteorológicas. Para realizar a análise espaço-temporal foram utilizadas imagens do Satélite Landsat 5 e Landsat 8, adquiridas gratuitamente no Instituto Nacional de Pesquisas Espaciais (INPE) entre os meses de junho e outubro. Os dados meteorológicos (temperatura máxima média, umidade relativa e precipitação total) foram obtidos no site do Instituto Nacional de Meteorologia (INMET). No período de estudo houve um decréscimo de $15,90 \%$ da vegetação nativa, e simultaneamente ocorreram alterações no microclima, com aumento de $2,06^{\circ} \mathrm{C}$ na temperatura e diminuição de $25 \%$ da umidade relativa. A progressiva pressão sobre a vegetação nativa para uso e ocupação do solo causou rápidas alterações na paisagem natural e no microclima da região.
\end{abstract}

\begin{abstract}
A B S T R A C T
The pressure on natural resources for agriculture and livestock activities has caused changes and losses of ecosystem services essential to the well-being of populations. The aim of this study was to evaluate the influence of deforestation on climate change in areas of agriculture and livestock exploitation in the city of Currais-PI. A space time analysis of land use and land cover in the municipality was carried out over a 32-year time series (1986 to 2018), correlating with meteorological variables. To perform the space time analysis Landsat 5 and Landsat 8 satellite images were used, acquired free of charge from the National Institute for Space Research (INPE), between june and october. Meteorological data (mean maximum temperature, relative humidity and total precipitation) were obtained from the website of the National Institute of Meteorology (INMET). During the study period there was a decrease of $15.90 \%$ of the native vegetation and simultaneously changes in the microclimate, with a 2.06 ${ }^{\circ} \mathrm{C}$ increase in temperature and a $25 \%$ decrease in relative humidity. The progressive pressure on native vegetation for land use and land cover caused quick changes in the natural landscape and microclimate of the region.
\end{abstract}

\section{INTRODUÇÃO}

As florestas se destacam pela alta capacidade em prover serviços ecossistêmicos que contribuem para a manutenção das funções ecológicas e garantem o bem-estar humano no espaço e ao longo do tempo (COSTANZA et al., 2014; MUTOKO et al., 2015). Apesar da importância, a conversão dessas áreas florestais em campos agrícolas ou de pastagem têm provocado grandes alterações nas paisagens (SILVA et al., 2014).

A consequência disso é a perda e degradação das funções ecológicas essenciais à manutenção dessas florestas, principalmente, o potencial em absorver continuamente $\mathrm{CO}_{2}$ da atmosfera e depositar em forma de carbono (C) em sua biomassa, o que contribui com diminuição dos efeitos das

\begin{tabular}{lc}
\hline Revista Verde & v. 14, n.4, out.-dez, p.540-545, 2019 \\
ISSN 1981-8203 & doi: $10.18378 /$ rvads.v14i4.6641 \\
Pombal, Paraíba, Brasil &
\end{tabular}


mudanças climáticas (MEA, 2005; GONZALES et al., 2014). No contexto atual das alterações climáticas tem havido uma preocupação em estudar o efeito do desmatamento na perda dos muitos serviços oferecidos pelas florestas (MIURA et al., 2015; BIRDSEY, 2015). Globalmente, estima-se uma perda média ponderada de 9,2\% no valor anual dos serviços ecossistêmicos provenientes da degradação da terra (SUTTON et al., 2016).

No Brasil, há uma redução estimada de $6,7 \%$ ao ano (SUTTON et al., 2016), tendo como principais causas das perdas substanciais desses serviços as mudanças na cobertura do solo e a diminuição dos ecossistemas nativos. Dentre esses ecossistemas florestais, destaca-se o bioma Cerrado que ao longo dos anos tem sofrido constantes ameaças por ter mais de $70 \%$ de sua cobertura vegetal original transformada, resultado decorrente da exploração agropecuária (REYDON; MONTEIRO, 2006).

A contínua ocupação dos solos do Cerrado justifica-se pelas características edáficas favoráveis à mecanização e a maior quantidade de incentivos fiscais para o uso da terra (LIMA et al., 2014; FRANÇA et al., 2017). O estado do Piauí possui $70 \%$ de sua extensão territorial coberta pela vegetação do Cerrado, da qual, 3 milhões de hectares estão aptas para cultivo e possui um cenário de grande potencial para expansão agropecuária (REYDON; MONTEIRO, 2006; SILVA et al., 2014).

Estas áreas localizam-se, geograficamente, em distintos pontos do Estado, mas nas últimas três décadas, a região sul do Piauí, teve um aumento significativo da atividade, ocasionando à expansão das chamadas fronteiras agrícolas (FRANÇA et al., 2017). No estado, 24 municípios se destacam na produção agrícola, principalmente o cultivo da soja (SANTOS et al., 2017). Dentre estes, inclui-se o município de Currais-PI, que nos últimos anos passou por um rápido processo de uso e ocupação da terra sem nenhuma preocupação com a aplicação de políticas públicas de conservação dos ecossistemas florestais, responsáveis pelo fornecimento dos muitos serviços ecossistêmicos essenciais ao bem-estar da população local.

Ante o exposto, objetivou-se avaliar a influência do desmatamento sob as variáveis climáticas em áreas de exploração agropecuária do município de Currais-PI.

\section{MATERIAL E MÉTODOS}

A pesquisa foi realizada no município de Currais-PI, que compreende uma área de $3.156,6 \mathrm{~km}^{2}$, stuado na região Sudoeste do Piauí, na microrregião do Alto medio Gurguéia. Essa região é economicamente ativa no estado em virtude de grandes projetos agrícolas, sobretudp, pelo predomínio do cultivo da soja. (Figura 1).

O clima da região, segundo a classificação de Köppen é AW' (caracterizado como quente e semi-úmido) com temperaturas superiores a $18^{\circ} \mathrm{C}$ (ALVARES et al., 2013). A precipitação pluviométrica média anual é definida no Regime Equatorial Continental, com isoietas anuais em torno de 700 a $1.200 \mathrm{~mm}$ e período chuvoso estendendo-se de novembro a maio, sendo os meses de janeiro, fevereiro e março, considerado o trimestre mais úmido (IBGE, 2000; PRAGANA et al., 2012).

A cobertura vegetal caracteriza-se por formações vegetacionais de dois biomas: Cerrado e Caatinga e em menor

proporção em área ecotonal (cerrado-caatinga) (CEPRO, 1996; BOTREL et al., 2015).

Figura 1. Localização geográfica do município de Currais, Piauí, Brasil.

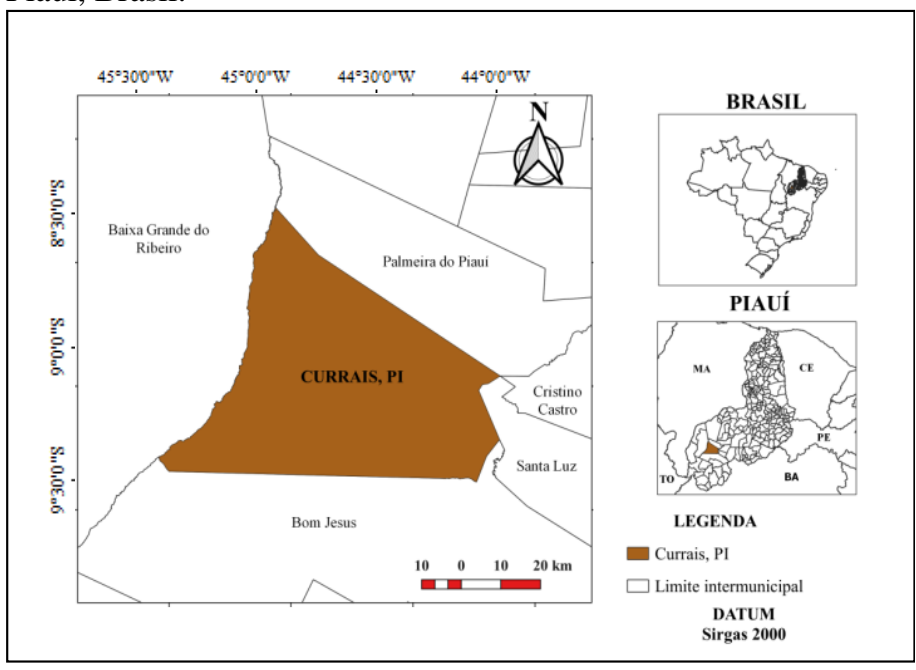

Para realização deste trabalho foram utilizadas imagens obtidas a partir do Satélite Landsat 5 sensor TM (Thematic Mapper), referentes aos anos de 1986, 1995 e 2005 e Landsat 8, que possui o sensor ótico OLI "Operational Land Imager" e o sensor infravermelho termal TIRS "Thermal Infrared Sensor", referente aos anos de 2015 e 2018, com resolução espacial de $30 \mathrm{~m}$, da órbita 220 e ponto 066 . As imagens utilizadas foram adquiridas gratuitamente no site eletrônico do INPE (Instituto Nacional de Pesquisas Espaciais) entre os meses de junho e outubro, período em que não há muitas nuvens que se caracterizariam como ruídos nas imagens (SEVER et al., 2012).

O processamento das imagens foi no software QGIS versão 3.4.1, utilizando as bandas 3,4 e 5 para realizar a composição colorida RGB com a seguinte configuração: R região do infravermelho próximo (banda 5); G - região vermelho (banda 4); e B - região do verde (banda 3). O sistema de referência adotado na pesquisa foi o SIRGAS 2000, utilizando Sistema Universal Transversa de Mercator (UTM).

A classificação das imagens foi utilizando o método do algoritmo da máxima verossimilhança, em que a identificação do objeto é feita pelas características pontuais por amostragem (acima de 200 pontos) considerando quatro classes fornecidas por Alvarenga Neto (2009) e levando em consideração as especificidades do município (Tabela 1).

Tabela 1. Classes usadas na caracterização supervisionada das imagens, Currais, Piauí, Brasil.

$$
\text { Classes }
$$

Formação Florestal

Agropecuária

Corpos hídricos

Área não vegetada Descrição

Áreas com vegetação nativa de domínio Cerrado, Caatinga e adjacentes aos cursos d'água.

Áreas destinadas às atividades agrícolas (Soja, milho, sorgo e etc.) e pastagem.

Rios, açudes, barragens, lagos e outros.

Área com urbanização, rodovias e estradas, área de encosta, entre outros. 
Após a classificação das imagens foram obtidos os valores em termos percentuais (\%) de cada uma das classes e o correspondente valor representado em $\mathrm{km}^{2}$.

Os dados meteorológicos foram obtidos no BDMEP Banco de Dados Meteorológicos para Ensino e Pesquisa, disponíveis no Instituto Nacional de Meteorologia-INMET (INMET, 2019). As variáveis climáticas avaliadas foram: temperatura máxima média $\left({ }^{\circ} \mathrm{C}\right)$, umidade relativa do ar $(\%)$ e precipitação total $\left(\mathrm{mm}^{\mathrm{anno}} \mathrm{p}^{-1}\right)$. Foram considerados os dados absolutos, informados para o intervalo entre 1986 e 2018, da estação meteorológica (82975) mais próxima da área de estudo, localizada no município de Bom Jesus-PI, distante $8 \mathrm{~km}$ de Currais-PI.

\section{RESULTADOS E DISCUSSÃO}

Ao analisar a dinâmica do uso e ocupação dos solos no município de Currais-PI, foi observado que não houve alteração ao longo dos anos para as áreas não vegetadas (urbanização, rodovia, corpos hídricos e etc), permanecendo em menos de $1 \%$ do município (Figura 2). A formação florestal foi à classe que apresentou maior percentual de cobertura entre os anos de 1986 e 2018, apresentando $99 \%$ da área do município no ano de 1986.

Figura 2. Dinâmica espaço-temporal da cobertura do solo no município de Currais, Piauí, Brasil.

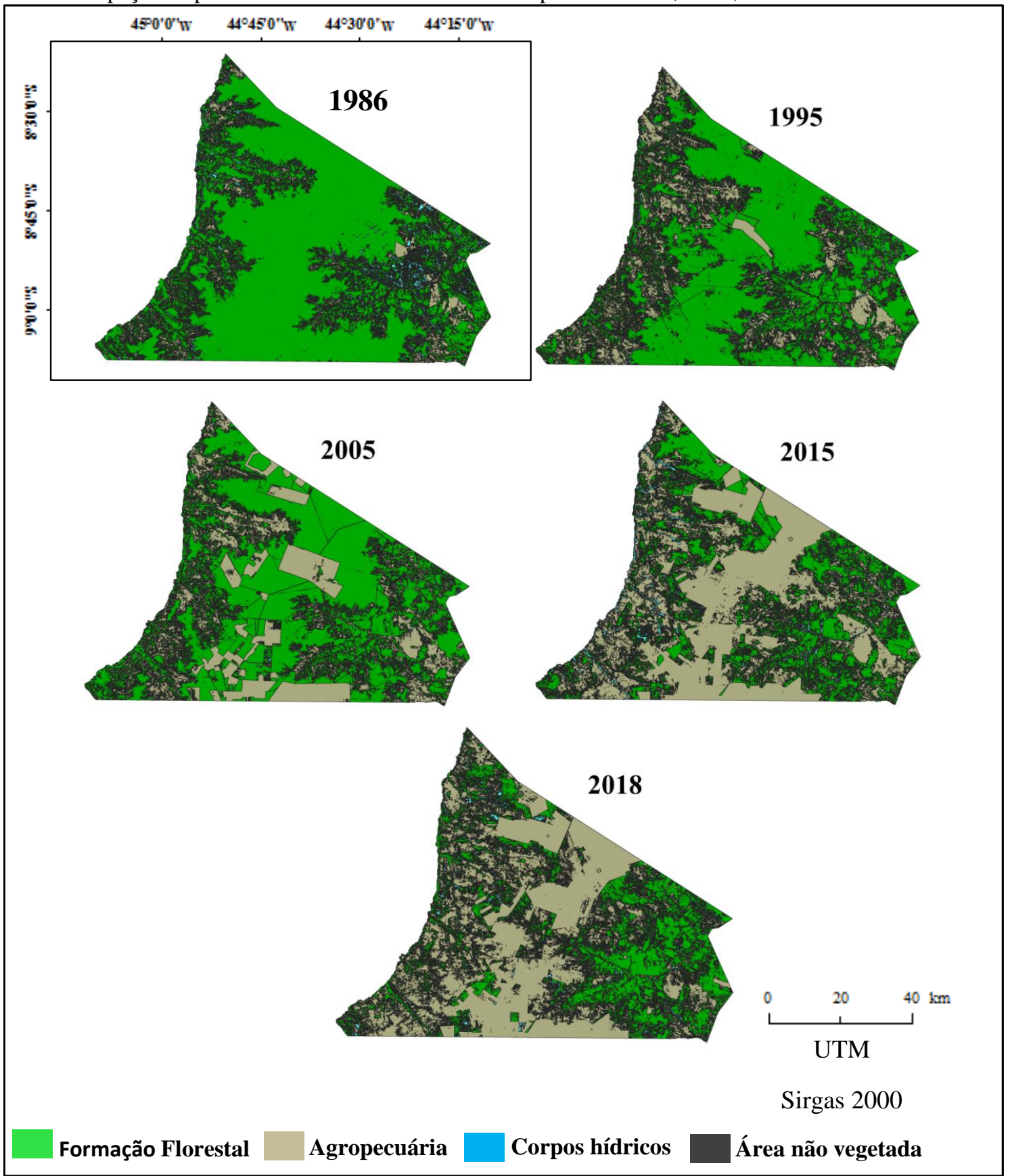


No entanto, a partir do ano de 1995 iniciou-se uma intensa transformação da paisagem natural, apresentando os primeiros indícios do uso e ocupação da terra por atividade agrícola (Figura 2). Aguiar e Monteiro (2005) mencionam que a década de 1990 é tida como ponto de partida para a implementação e intensificação de grandes projetos voltados para o agronegócio, principalmente o cultivo da soja.

A expansão da agropecuária na região em estudo teve um aumento expressivo, passando de 0,06\% (194,89 ha) em 1995, para $16 \%$ (50.772,63 ha) no ano de 2018 . Por outro lado, devido a esse avanço, neste mesmo período houve simultaneamente um decréscimo da cobertura vegetal nativa em 15,90\%. Essa tendência também tem sido diagnosticada em áreas adjacentes ao município de Currais-PI, com perda de até 54,81\% da vegetação nativa e um aumento de até $297,98 \%$ de solo exposto entre os anos de 1984 e 2015 (FRANÇA et al., 2017; SANTOS et at., 2017).

A contínua ocupação e uso dos solos nessas regiões são justificados principalmente pelas características edáficas favoráveis à mecanização, propriedades com valores abaixo do mercado, incentivos fiscais concedidos pelo governo para o uso da terra, além do esgotamento de terras em outras regiões do país e o elevado preço da soja no mercado internacional (BORGHI et al., 2014; FRANÇA et al., 2017).

A expansão do agronegócio e a intensa pressão sobre os recursos naturais têm causado preocupações com as possíveis perdas de serviços essenciais à manutenção dos ecossistemas e ao bem-estar das populações residentes nessa região, como por exemplo, as mudanças climáticas que de acordo com Santos et al. (2017), vem ocorrendo na região com redução na precipitação de até $172,08 \mathrm{~mm}^{-a n o}{ }^{-1}$; aumento da temperatura máxima em $0,91^{\circ} \mathrm{C}$ e diminuição da umidade relativa do ar em $7,43 \%$.

Com relação às mudanças climáticas observa-se um aumento significante da temperatura máxima média $\left({ }^{\circ} \mathrm{C}\right)$ no decorrer do período de 32 anos analisado, passando de $32,13^{\circ} \mathrm{C}$ em 1986 para $34,19^{\circ} \mathrm{C}$ no ano de 2018 , configurando em uma elevação de $2,06^{\circ} \mathrm{C}$ na temperatura média (Figura 3 ).

Figura 3. Dinâmica do uso e ocupação do solo e temperatura máxima média $\left({ }^{\circ} \mathrm{C}\right)$ no município de Currais, Piauí entre 1986 e 2018.

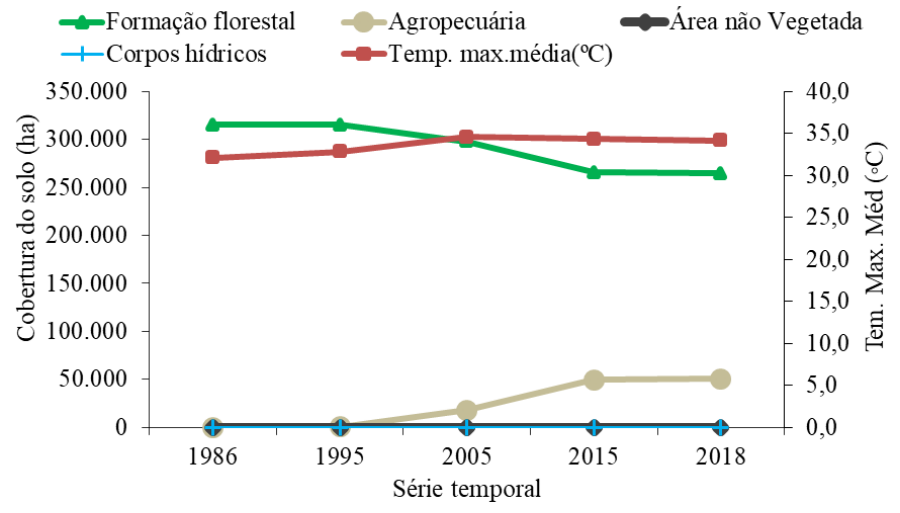

A umidade relativa do ar (UR) apresentou decréscimo no intervalo entre as décadas estudadas (1986 a 2018), com declínio de 25,75\% (Figura 4). A precipitação total permaneceu praticamente inalterada entre os anos avaliados, com $986,5 \mathrm{~mm}$ $\mathrm{ano}^{-1} \mathrm{em} 1986$ e $985,5 \mathrm{~mm}$ ano $^{-1} \mathrm{em} 2018$.

Figura 4. Precipitação total e umidade relativa média no município de Currais-Piauí entre 1986 e 2018.

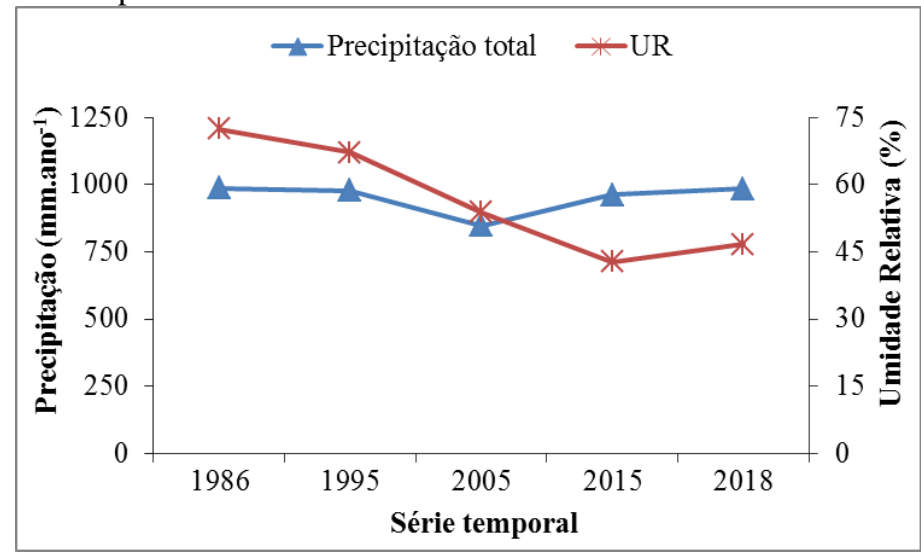

O aumento na temperatura e diminuição da UR esta diretamente relacionada com o inicio do desmatamento das áreas de vegetação nativa na região (Figura 2). Esses resultados também foram corroborados por Albuquerque et al. (2016) ao verificarem que as mudanças na cobertura vegetal têm influência direta sobre a variação da temperatura e umidade relativa do ar.

Essas variações microclimáticas estão fortemente relacionadas ao uso e ocupação do solo, ocasionando uma situação ambiental desconfortável para aqueles que habitam ou circulam nessas áreas (GHENO et al., 2012).

Esses resultados refletem a importância de manter as áreas de florestas destinadas pela lei 12.651/12 (BRASIL, 2012) como reservas legais (RL) e áreas de preservação permanente (APPs) desempenhando suas funções ecológicas, como por exemplo, a evapotranspiração pelas plantas, que exerce efeito positivo, absorvendo calor e melhorando as condições de conforto térmico, contribuindo para a melhoria da qualidade de vida e o bem-estar das populações (ABREU-HARBICH et al., 2015).

\section{CONCLUSÕES}

A progressiva pressão sobre a vegetação nativa no município de Currais-PI, para uso e ocupação do solo pela atividade agropecuária causa alterações na paisagem natural e no microclima da região.

A vegetação nativa no período de estudo teve um decréscimo de $15,90 \%$, e simultaneamente ocorreram alterações no microclima, com aumento de $2,06^{\circ} \mathrm{C}$ na temperatura e diminuição de $25 \%$ da umidade relativa.

\section{AGRADECIMENTOS}

Agradecemos ao Instituto Nacional de Pesquisas Espaciais-INPE e ao Instituto Nacional de MeteorologiaINMET pela disponibilidade dos dados. 


\section{REFERÊNCIAS}

AGUIAR, T. J. A.; MONTEIRO, M. do S. L. Modelo Agrícola e Desenvolvimento Sustentável: a ocupação do Cerrado Piauiense. Ambiente \& Sociedade, v. 8, n. 2, 2005.

ALBUQUERQUE, M. M.; LOPES, W. G. R. Influência da vegetação em variáveis climáticas: estudo em bairros da cidade de Teresina, Piauí. Revista Ra'eGa, Curitiba, v. 36, p. 38-68, 2016. 10.5380/raega.v36i0.39719

ALVARES, C. A.; STAPE, J. L.; SENTELHAS, P. C.; GONÇALVES, J. L. M.; SPAROVEK, G. Köppen's climate classification map for Brazil. Meteorologische Zeitschrift, v. 22, p. 711-728, 2013. 10.1127/0941-2948/2013/0507

ABREU-HARBICH, L.V,; LABAKI, L. C.;; MATZARAKIS, A. Effect of tree planting design and tree species on human thermal comfort in the tropics. Landscape and Urban Planning, v. 1, p. 111-131, 2015. 10.1016/j.landurbplan.2015.02.008

ALVARENGA NETO, I. Uso de Ferramentas de SIG e Sensoriamento Remoto para o monitoramento do desmatamento em Unidades de Conservação: Estudo de caso da Floresta Nacional do Bom Futuro-RO. 2009. 110 f. Dissertação (Mestrado em Geografia) - Universidade Federal de Rondônia, Porto Velho, 2009.

BIRDSEY, R.; PAN, Y. Trends in management of the world's forests and impacts on carbon Stocks. Forest Ecology and $\begin{array}{llll}\text { Management, } & \text { v.355, } & \text { p.83-90, } & \end{array}$ 10.1016/j.foreco.2015.04.031

BORGHI, E.; BORTOLON, L.; AVANZI, J. C.; BORTOLON, E. S. O.; UMMUS, M. E.; GONTIJO NETO, M. M.; COSTA, R. V. Desafios das novas fronteiras agrícolas de produção de milho e sorgo no Brasil: desafios da região do MATOPIBA. In: KARAM, D.; MAGALHÃES, P. C. (Ed.). Eficiência nas cadeias produtivas e o abastecimento global. Anais, Sete Lagoas: ABMS, 2014. cap. 25, p. 263-278.

BOTREL, R.T.; BRITO, D. R. S.; SOUSA, W.C.; SOUZA, A.M.; HOLANDA, A.C. Phenology of tree species in Caatinga/Cerrado boundary in Southern Piauí. Rev. Verde de Agroecologia e Desenvolvimento Sustentavel, v.10, n.3, p.0712, 2015. 10.18378/rvads.v10i3.3587

BRASIL. Lei Federal no. 12.651, de 25 de Maio de 2012. Dispõe sobre a proteção da vegetação nativa, Brasília, Brasil, $2012 . \quad$ Disponível em:

<http://www.planalto.gov.br/ccivil_03/_ato20112014/2012/lei/l12651.htm>

CEPRO. Piauí: Caracterização do quadro natural. Teresina, PI: Fundação CEPRO, 1996. 116p.

COSTANZA, R.; DE GROOT, R.; SUTTON, P.; DER PLOEG, S.V.; ANDERSON, S.J.; KUBISZEWSKI, I.; FARBER, S.; TURNER, R.K. Changes in the global value of ecosystem services. Global Environmental Change, v. 26, p. 152-158, 2014. 10.1016/j.gloenvcha.2014.04.002

FRANÇA, L. C. J.; RIBEIRO, N. M. A. R.; SANTOS, E. L.; NORONHA, F.C.C.; SANTOS, E.L.; RIBEIRO, A. T. Caracterização da cobertura vegetal e uso do solo no município de Uruçuí, Piauí, Brasil. Nativa, v. 5, p. 337-341, 2017. $\underline{10.5935 / 2318-7670 . v 05 \mathrm{n} 05 \mathrm{a} 06}$

GHENO, E. L.; FRANÇA, M.S.; MAITELLI, S.F. Variações microclimáticas na área urbana de Sinop no final da estação chuvosa. Revista Educação. Cultura e Scuola, v. 2, n.1, p. 139153, 2012.

GONZALEZ, P.; KROLL, B.; VARGAS, C.R. Tropical rainforest biodiversity and aboveground carbon changes and uncertainties in the Selva Central, Peru. Forest Ecology and Management, v.312 , p.78-91, 2014. 10.1016/j.foreco.2013.10.019

INSTITUTO BRASILEIRO DE GEOGRAFIA E ESTATÍSTICAS (IBGE). Censo Demográfico 2000: Características da população e dos domicílios. Rio de Janeiro: IBGE, 2000.

INMET. Instituto Nacional de Meteorologia. "Banco de Dados para Pesquisa e Ensino - BDMEP. 2019". Disponível em: <http://www.inmet.gov.br/portal/> . Acesso em: 25 de agosto de 2019.

LIMA, T. P.; FRANÇA, L. C. J.; SILVA, J. B. L.; FURTINI, M. B. Intensidade de Exploração do Solo em uma Sub-Bacia do Rio Uruçuí-Preto, Piauí. Revista Educação Agrícola Superior, Brasília, v. 29, n. 1, 2014. 10.12722/0101-756X.v29n01a02

MILLENNIUM ECOSYSTEM ASSESSMENT (MEA). Ecosystems and Human Well- Being: Synthesis. Island Press, DC. 2005.

MIURA, S.; AMACHER, M.; HOFER, T.; SAN-MIGUELAYANZ, J.; ERNAWATI.; THACKWAY, R. Protective functions and ecosystem services of global forests in the past quarter-century, Forest Ecology and Management, v.352, p. 3546, 2015. 10.1016/j.foreco.2015.03.039

MUTOKO, M.C.; HEIN, L.; SHISANYA, C.A. Tropical forest conservation versus conversion trade-offs: Insight from analysis of ecosystem services provided by Kakamega rainforest in Kenya. Ecosystem Services, v. 14, p.1-11, 2015. 10.1016/j.ecoser.2015.03.003

PRAGANA, R. B.; RIBEIRO, M. R.; NÓBREGA, J. C. A.; RIBEIRO FILHO, M. R.; COSTA, J. A. Qualidade física de Latossolos Amarelos sob plantio direto na região do cerrado piauiense. Revista Brasileira de Ciência Solo, v. 36, n. 5, p. 1591-1600, 2012. 10.1590/S0100-06832012000500023.

REYDON, B. P.; MONTEIRO, M. S. L. A ocupação do cerrado piauiense: um processo de valorização fundiária. REYDON, B. 
P.; CORNÉLIO; F.N.M (Orgs). Mercados de terras no Brasil: estrutura e dinâmica. Brasília: NEAD, p. 95-122. 2006.

SANTOS, J. C.; LISBOA, G. S.; FRANÇA, L. C. J.; STEPKA, T. F. ; SILVA, J. B. L.; MIRANDA, D. L. C.; CERQUEIRA, C. L. Relação entre variáveis meteorológicas e o uso e cobertura do solo no Sudoeste do Piauí, Brasil. Nativa, v. 5, p. 414-420, 2017. 10.5935/2318-7670.v05n06a06

SEVER, L.; LEACH, J.; BREN, L. Remote sensing of post-fire vegetation recovery; a study using Landsat 5 TM imagery and NDVI in North-East Victoria. Journal of Spatial Science, v. 57, n. 2 , p. $175-191,2012$. 10.1080/14498596.2012.733618

SILVA, J. B. L.; FERREIRA, W. L.; ALMEIDA, K. N. S.; SOUZA, K. B.; NOBREGA, J. C. A. Evolução temporal do desmatamento e expansão agrícola entre 1984 a 2010 na subbacia do Rio Uruçuí-Preto, Piauí. Engenharia na Agricultura, v. 22, n. 3, p. 254-261, 2014. 10.13083/1414-3984.v22n03a08

SUTTON, P.C.; ANDERSON, S.J.; COSTANZA, R.; KUBISZEWSKI, I. The ecological economics of land degradation: Impacts on ecosystem service values, Ecological $\begin{array}{llll}\text { Economics, } & \text { v.129, } & \text { p.182-192, } & 2016 .\end{array}$ $\underline{10.1016 / j . e c o l e c o n .2016 .06 .016}$ 